\title{
FURTHER RESULTS ON PRIME ENTIRE FUNCTIONS
}

\author{
BY \\ FRED GROSS AND CHUNG-CHUN YANG
}

\begin{abstract}
Let $H$ denote the set of all the entire functions $f(z)$ of the form: $f(z) \equiv h(z) e^{p(z)}+k(z)$ where $p(z)$ is a nonconstant polynomial of degree $m$, and $h(\neq 0)$, $k(\not$ constant $)$ are two entire functions of order less than $m$. In this paper, a necessary and sufficient condition for a function in $\boldsymbol{H}$ to be a prime is established. Several generalizations of known results follow. Some sufficient conditions for primeness of various subclasses of $H$ are derived. The methods used in the proofs are based on Nevanlinna's theory of meromorphic functions and some elementary facts about algebraic functions.
\end{abstract}

I. Introduction. According to [10], an entire function $F(z)=f(g(z))$ is said to have $f(z)$ and $g(z)$ as left and right factors respectively, provided that $f(z)$ is meromorphic and $g(z)$ is entire ( $g$ may be meromorphic when $f$ is rational). $F(z)$ is said to be prime (pseudo-prime) if every factorization of the above form implies that one of the functions $f$ or $g$ is bilinear (a rational function). $F$ is said to be $E$-prime ( $E$-pseudo-prime) if every factorization of the above form into entire factors implies that one of the functions $f$ or $g$ is linear (a polynomial). We say that two entire function $F_{1}$ and $F_{2}$ are relatively $R$-polynomial prime if and only if the only possible common polynomial right factors of $F_{1}$ and $F_{2}$ are linear functions.

Although the subject of factorization has been studied extensively by many authors such as Fatou [3], Ritt [15], Rosenbloom [16], Baker [1], Yang [10], [17], Gross [1], [7], [8], [9], [10], Ozawa [13] and Goldstein [4], [5], [6], only a few classes of entire functions are known to be prime or pseudo-prime. We exhibit some of these classes below and proceed to enlarge them.

Theorem A (Baker-Gross [1]). Every entire function of the form $e^{z}+p(z)$ where $p(z)$ is a nonconstant polynomial is E-prime.

Theorem B (Goldstein [4]). Every entire function $F$ of finite order with $\delta(a, F)$ $=1$ for some $a \neq \infty$, where $\delta(a, F)$ denotes the Nevanlinna deficiency is $E$-pseudoprime.

Remark. Goldstein remarks at the end of [4] that " $F$ is pseudo-prime" can be proved, and a proof of this is given in [6].

In $[10, p .213]$ the present authors indicated that Theorem A can be generalized as follows.

Received by the editors December 22, 1972 and, in revised form, February 16, 1973.

AMS (MOS) subject classifications (1970). Primary 30A64, 30A20; Secondary 30A66.

Key words and phrases. Factorization, factor, entire function, meromorphic function, prime function, pseudo-prime function, $R$-polynomial prime, Nevanlinna theory, $E$-prime.

Copyright 0 1974, American Mathematical Society 
Theorem C. Let $h_{1}(z)$ be a nonzero polynomial, and $h_{2}(z)$ ( $\not \equiv$ constant) be an entire function of order less than the degree of the polynomial $p(z)$. Then the only possible factorization of $h_{1}(z) e^{p(z)}+h_{2}(z)$ is of the form $h_{1}(z) e^{p(z)}+h_{2}(z)$ $=f(Q(z))$, where $Q(z)$ is a polynomial of degree no greater than the degree of $p(z)$.

Remark. This result is a special case of Lemma 4 in this paper.

If $p(z) \equiv z$, Goldstein [5] obtained the following stronger result.

Theorem D. If $q, h(q \not \equiv 0, h \not \equiv$ constant) are entire functions of order less than one, then $q(z) e^{z}+h(z)$ is E-prime.

In this paper, we combine methods used in [10] and some elementary facts about algebraic functions to generalize Theorems $\mathbf{C}$ and $\mathrm{D}$ substantially. We consider the class $H$ defined as the set of all entire functions of the form $h(z) e^{p(z)}+k(z)$, where $p(z)$ is a nonconstant polynomial of degree $m$, and $h(\not \equiv 0)$ and $k$ ( $\not \equiv$ constant) are two entire functions of order less than $m$. We have obtained a necessary and sufficient condition for a function in $H$ to be prime and also derived some sufficient conditions for various subclasses of $H$ to be prime functions.

Throughout this paper, it is assumed that the reader is familiar with the usual symbols of the Nevanlinna theory of meromorphic functions: $T(r, f), N(r, f)$, etc. (see e.g. [11]). We recall here that the order $\rho$ of a meromorphic function $f$ is defined as $\rho=\varlimsup_{r \rightarrow \infty} \log T(r, f) / \log r$ and note that for a polynomial $p(z)$ of degree $m$ the order of the entire function $e^{p(z)}$ is $m[11$, p. 7].

II. Main results.

2.1. Necessary conditions.

Theorem 1. Let $p(z)$ be a nonconstant polynomial of degree $m, h(z)(\not \equiv 0)$ and $k(z)\left(\not \equiv\right.$ constant) be two entire functions of order less than $m$. Then $h(z) e^{p(z)}+k(z)$ is either prime or it can only be factorized as:

$$
h(z) e^{p(z)}+k(z)=f(L(z)),
$$

where $L(z)$ is a nonlinear polynomial of degree $n$, and $f(z)=\mu(z) \exp \left(c[L(z)]^{d}\right)$ $+\beta(z)$ an entire function, with the following three relations being satisfied:

(i) $n \mid m$ (i.e. $m / n$ is an integer),

(ii) $h(z) e^{p(z)} \equiv \mu(L(z)) \exp \left(c[L(z)]^{d}\right)$, where $d=m / n$ an integer, $c$ is a constant, and $\mu(z)$ is an entire function of order less than $d$, and

(iii) $k(z)=\beta(L(z))$ where $\beta(z)$ is an entire function of order less than $d$.

Remark. The example $\exp \left(k z^{m}\right)+1=\left(z^{k}+1\right) \circ \exp \left(z^{m}\right), m \geq 1, k>1$, shows that the restriction on $k \not \equiv$ constant in the theorem is necessary.

Remarks. (A) Suppose that equation (1) holds. Then it follows from assertions (ii) and (iii) that $h e^{p}$ and $k$ have a nonlinear polynomial $L$ as their common right factor, and hence $h e^{p}$ and $k$ are not $R$-polynomial prime. 
(B) From assertion (i), it follows particularly that $n \leq m$, i.e., the degree of $L$ is no greater than the degree of $p$. This observation is very useful in our applications. For instance, taking $p(z) \equiv z$ in the theorem, we conclude immediately that $h e^{z}+k$ does not possess any nonlinear polynomial right factor. This and Lemma 4 (see $\$ 5$ below) yields a generalization of Theorem $C$.

2.2. A necessary and sufficient condition and some sufficient conditions.

Theorem 2. Let $p(z)$ be a nonconstant polynomial of degree $m, h(z)$ and $k(z)$ be two entire functions of order less than $m$ and $h \not \equiv 0, k \not \equiv$ constant. Then $h e^{p}+k$ is prime if and only if he $e^{p}$ and $k$ are relatively $R$-polynomial prime.

Application. $h e^{p}+z$ is prime for any polynomial $p(z)$ and any entire function $h$ of order less than the degree of $p$.

Corollary 2.1. Let $p, h, k$ be as in Theorem 2. Then $h e^{p}+k$ is prime if he possesses no nonlinear polynomial right factors.

Application. $q(z) e^{p(z)}+k(z)$ is prime for any nonconstant polynomial $p, k$ any entire function of order less than the degree of $p$, and $q(z)$ any polynomial of prime degree.

Corollary 2.2. Let $p, h, k$ be as in Theorem 2. Assume further that $k$ is transcendental and prime. Then he $e^{p}+k$ is prime.

Application. $z^{4} e^{z^{2}}+e^{z}+z$ is prime.

Corollary 2.3. Let $p, h, k$ be as in Theorem 2. Then $h e^{p}+k$ is prime if he $p / k$ does not possess any factorization which consists of a meromorphic left factor and a nonlinear polynomial right factor.

Application. $\alpha(z) h(z) e^{p(z)}+z \alpha(z)$ is prime for any polynomial $p(z)(\not \equiv$ constant) and any two nonzero entire functions $\alpha$ and $h$ of order less than the degree of $p$. Since $\alpha h e^{p}+z \alpha \equiv \alpha\left(h e^{p}+z\right)$ and $h e^{p}+z$ is prime, we thus have a very interesting phenomenon that there exists a prime function $f$ such that $\alpha f$ remains prime for any entire function $\alpha(\not \equiv 0)$ with order less than that of $f$.

2.3. On the zeros of $h$. As a variation, we obtain the following criterion in terms of the geometric configuration of the zeros of $h$.

Corollary 2.4. Let $p, h, k$ be as in Theorem 2. Assume further that $h$ has infinitely many zeros and all but finitely many of the zeros are real (or asymptotic to any straight line). Then he $e^{p}+k$ is prime if $k(z) \not \equiv k(-z+c)$ for any constant $c$.

Proof. It is easy to verify that the hypotheses imply that the only possible polynomial right factors of $h e^{p}$ are quadratics. The assertion follows from this and Theorem 1.

Application. $(\cos z) e^{p(z)}+q(z)$ is prime for any polynomial $p$ of degree $\geq 2$ and any polynomial $q(z)$ of odd degree. This result remains valid when $p(z)=r z+b$, $r$ rational [7]. The authors conjecture that it remains true for arbitrary complex $r$. The methods of this paper, however, do not seem to yield this result. 
2.4. On the relationships between $p$ and $k$. The following result follows immediately from assertions (i) and (iii) of Theorem 1 and Lemma 4.

Theorem 3. Let $p$ be a nonconstant polynomial of degree $m$ and let $h(\not 0)$ and $k$ ( $\equiv$ constant) be two entire functions of order less than $m$. If $k$ has no nonlinear polynomial factors whose degree divides $m$ then $h e^{p}+k$ is prime.

Corollary 2.5. Let $p$ and $h$ be as in Theorem 3. Let $k$ be any nonconstant entire function of order less than $m$ and let $h_{1}$ be an entire function of order less than $m$ and $q(z)$ be a polynomial of degree less than $m$. Suppose that $p$ and $q$ are relatively $R$ polynomial prime. Then $h(z) e^{p(z)}+h_{1} e^{q(z)}+k$ is prime.

We note that the only possible nonlinear polynomial right factor for a periodic and entire function is a polynomial of degree two (see e.g. [14] or [1, p. 33]).

Hence, we have the following:

Corollary 2.6. Let $p, h, k$ be as in Theorem 3. Assume further that the degree of $p$ is odd and that $k$ is a nonconstant periodic function. Then $e^{p}+k$ is prime.

Application. Given any entire and periodic function $H$ of finite order $\rho$, one can always find an entire function $\alpha$ such that $\alpha H$ is prime. We simply choose $h \equiv k \equiv H$ and $p$ to be any polynomial of degree $m$ with $m$ odd and $>\rho$.

Theorem 4. Let $p, h, k$ be as in Corollary 2.5. Assume further that the order of $h$ is less than one. Then $h^{p}+k$ is prime if and only if $p$ and $k$ are relatively $R-$ polynomial prime.

Remark. The order restriction on $h$ is necessary. For if we allow $h$ to be of order greater than or equal to one, then the assertion may fail to be true. For instance, $e^{z} e^{z^{2}-z}+z^{2}$ is not prime (in this example, $h=e^{z}, p=z^{2}-z$, and $k=z^{2}$ ).

III. Preliminaries. Before going into the proofs of the theorems, we discuss some lemmas which are needed later.

Lemma $1\left[12\right.$, p. 267]. Let $p(z, \omega) \equiv z^{n}+a_{1} z^{n-1}+a_{2} z^{n-2}+\cdots+a_{n}-\omega$ where $a_{i}(i=1,2, \ldots, n)$ are constants, and write $p(z, \omega) \equiv \prod_{i=1}^{n}\left(z-z_{i}(\omega)\right)$. Then every elementary symmetric function in $z_{i}(\omega)$ is a polynomial in $\omega$.

Lemma 1 enables us to establish the following more general result.

Lemma 2. Let $\omega, z_{i}(\omega)(i=1,2, \ldots, n)$ be as in Lemma 1 , and $g(z)$ be an entire function. Then $\sum_{i=1}^{n} g\left(z_{i}(\omega)\right)$ is an entire function in $\omega$.

Proof. Let us write the Taylor series for $g$ as

$$
g(z)=a_{0}+a_{1} z+a_{2} z^{2}+\cdots=\sum_{j=0}^{\infty} a_{j} z^{j}
$$

Then in any finite domain we have

$$
\sum_{i=1}^{n} g\left(z_{i}(\omega)\right)=\sum_{i=1}^{n} \sum_{j=0}^{\infty} a_{j}\left[z_{i}(\omega)\right]^{j} .
$$


Since the infinite series is convergent absolutely in every finite domain, we can rearrange the double summation and get

$$
\sum_{i=1}^{n} g\left(z_{i}(\omega)\right)=\sum_{j=0}^{\infty}\left(\sum_{i=1}^{n} a_{j}\left[z_{i}(\omega)\right]^{j}\right)=\sum_{j=0}^{\infty} p_{j}(\omega),
$$

where $p_{j}, j=0,1,2, \ldots$, are polynomials. The lemma is thus proved.

Lemma 3 (Gross [9, p. 568]). Let $F(z)$ be an entire function. Suppose that $F$ is not periodic, then $F$ is prime if and only if $F$ is $E$-prime.

This lemma enables us, from here on, to consider only entire factors since it is easy to verify that all the functions in $H$ are nonperiodic.

Lemma 4 (Goldstein [5, Theorem 6]. Let $m$ be a given positive integer, $p_{m}(z) a$ nonconstant polynomial of degree $m, \varphi_{m}(\not \equiv$ constant $), \psi_{m}(\not \equiv 0)$ entire functions of order less than $m$. Write $F(z)=\varphi_{m}(z)+\psi_{m}(z) \exp \left(p_{m}(z)\right)$ and suppose that $F(z)=f(g(z))$ with $f(z), g(z)$ nonlinear entire functions. Then $g(z)$ is a polynomial of degree $k$ and $f(z)$ is of order $\rho_{f}$ such that $k \rho_{f}=m$ and $k \leq m$.

Lemma 5 (see e.g., Gross [8, p. 4]). If $F, f$ are transcendental entire functions, $p$ is a polynomial such that $F=f(p)$, then the order of $F$ is equal to the product of the order of $f$ and the degree of $p$.

Remark. This result will be used implicitly throughout the rest of the paper.

IV. The proof of Theorem 1. Suppose that $F=h e^{p}+k$ is not prime; then by Lemma 4 the only possible factorization for $F$ is of form (1). Let

$$
\begin{array}{ll}
p(z) \equiv c_{0}+c_{1} z+c_{2} z^{2}+\cdots+c_{m} z^{m} & \left(c_{m} \neq 0\right), \\
L(z) \equiv a_{0}+a_{1} z+\cdots+a_{n} z^{n} & \left(a_{n} \neq 0\right),
\end{array}
$$

and

$$
\omega=L(z) .
$$

We have for sufficiently large $|\omega|$ (it will be assumed without loss of generality that all $\omega$ below are sufficiently large).

$$
L(z)-\omega \equiv a_{n}\left[z-z_{1}(\omega)\right]\left[z-z_{2}(\omega)\right] \cdots\left[z-z_{n}(\omega)\right],
$$

where $z_{i}(\omega)(i=1,2, \ldots, n)$ are $n$ distinct branches (since, clearly, $P(z, \omega) \equiv L(z)$ - $\omega$ is irreducible and of degree $n$ in $z$ ).

It is also clear that for $i=1,2, \ldots, n$ we have a development of the form

$$
z_{i}(\omega)=\omega_{i}^{1 / n}\left(b_{0}+b_{-1} \omega_{i}^{-1 / n}+b_{-2} \omega_{i}^{-1 / 2 n}+\cdots\right)
$$

valid in a neighborhood of $\infty$, where $\omega_{i}^{1 / n}=\rho_{i} \omega_{1}^{1 / n}, \rho_{i}(i=1,2, \ldots, n)$ are $n$ distinct roots of unity, $\rho_{1}=1$, and $\omega_{1}^{1 / n}$ is a fixed branch of $\omega^{1 / n}$. For $z_{i}(\omega)$ as above, we have 


$$
h\left(z_{i}(\omega)\right) \exp \left(p\left(z_{i}(\omega)\right)\right)+k\left(z_{i}(\omega)\right)=f(\omega), \quad i=1,2, \ldots, n .
$$

Thus for $i \neq 1$, the quotient

$$
\frac{h\left(z_{i}(\omega)\right) \exp \left(p\left(z_{i}(\omega)\right)\right)+k\left(z_{i}(\omega)\right)}{h\left(z_{1}(\omega)\right) \exp \left(p\left(z_{1}(\omega)\right)\right)+k\left(z_{1}(\omega)\right)}
$$

is always equal to one.

After dividing through the denominator and the numerator by $h\left(z_{1}(\omega)\right) \exp \left(p\left(z_{1}(\omega)\right)\right)$, the above quotient becomes

$\frac{\left(h\left(z_{i}(\omega)\right) / h\left(z_{1}(\omega)\right)\right) \exp \left(p\left(z_{i}(\omega)\right)-p\left(z_{1}(\omega)\right)\right)+\left(k\left(z_{i}(\omega)\right) / h\left(z_{1}(\omega)\right)\right) \exp \left(-p\left(z_{1}(\omega)\right)\right)}{1+\left(k\left(z_{1}(\omega)\right) / h\left(z_{1}(\omega)\right)\right) \exp \left(-p\left(z_{1}(\omega)\right)\right)}$.

If we substitute (9) into (5), then

$$
\begin{aligned}
p\left(z_{i}(\omega)\right)= & c_{m}\left[\omega_{i}^{1 / n}\left(b_{0}+b_{-1} \omega_{i}^{-1 / n}+b_{-2} \omega_{i}^{-1 / 2 n}+\cdots\right]^{m}\right. \\
& +c_{m-1}\left[\omega_{i}^{1 / n}\left(b_{0}+b_{-1} \omega_{i}^{-1 / n}+b_{-2} \omega_{i}^{-1 / 2 n}+\cdots\right]^{m-1}+\cdots+c_{0}\right. \\
= & d_{m} \omega_{i}^{m / n}+d_{m-1} \omega_{i}^{(m-1) / n}+\cdots+d_{0}+d_{-1} \omega_{i}^{-1 / n}+d_{-2} \omega_{i}^{-1 / 2 n}+\cdots,
\end{aligned}
$$

where $d_{m}(\neq 0), d_{i}(i=0, \pm 1, \pm 2, \ldots)$ are constants.

We are going to show that $n \mid m$. Assume that on the contrary $n \nmid m$. Then we choose a path of $\omega$, say $l$, a straight line in the $\omega$-plane tending to infinity such that, along $l, d_{m} \omega_{1}^{m / n}=\left|d_{m} \omega_{1}^{m / n}\right|$.

Now from (12),

$$
p\left(z_{i}(\omega)\right)-p\left(z_{1}(\omega)\right)=d_{m}\left(\rho_{i}^{m}-1\right) \omega_{1}^{m / n}+d_{m-1}\left(\rho_{i}^{m-1}-1\right) d_{1}^{(m-1) / n}+\cdots
$$

We note that if $n \nmid m$, then

$$
\operatorname{Re}\left(\rho_{i}^{m}-1\right)<0 \quad(i \neq 1) .
$$

We now examine the behavior of the functions $k\left(z_{1}(\omega)\right) / h\left(z_{1}(\omega)\right)$, $k\left(z_{i}(\omega)\right) / h\left(z_{1}(\omega)\right)$ along $l$. By assumption,. $h$ is an entire function of order less than $m$. Thus by Theorem 2.7.4 of $[2$, p. 22] on the behavior of the minimum modulus, we have $m_{h}(r) \neq O\left(\exp \left(-r^{m-\varepsilon}\right)\right.$ ) (for any $\varepsilon>0$, where $m_{h}(r)$ $\left.=\min _{|z|-r}\left|h\left(r e^{i \theta}\right)\right|\right)$. Using this fact, and the fact that $k$ is an entire function of order less than $m$, and noting that (14) implies that $\left|\exp \left(p\left(z_{i}(\omega)\right)-p\left(z_{1}(\omega)\right)\right)\right|$ grows much slower than $\exp \left(-\alpha|\omega|^{m / n}\right)$ as $\omega \rightarrow \infty$ for some $\alpha>0$, we conclude, after a simple verification, that the quantities $\left[h\left(z_{i}(\omega)\right) / h\left(z_{1}(\omega)\right)\right] \exp \left(-p\left(z_{1}(\omega)\right)\right)$, $\left[k\left(z_{1}(\omega)\right) / h\left(z_{1}(\omega)\right] \exp \left(-p\left(z_{1}(\omega)\right)\right)\right.$ and $\left[k\left(z_{i}(\omega)\right) / h\left(z_{1}(\omega)\right)\right] \exp \left(p\left(z_{i}(\omega)\right)-p\left(z_{1}(\omega)\right)\right)$ all tend to 0 as $\omega \rightarrow \infty$ through a suitable sequence $\left\{\omega_{n}\right\}$ on $l$. Thus, (11) must have a limit zero as $\omega_{n} \rightarrow \infty$, a contradiction. Hence, we must have $n \mid m$. $n \mid m$. 
Therefore, from (12), we have

$$
p\left(z_{i}(\omega)\right)=d_{m} \omega^{d}+d_{m-1} \omega_{i}^{(m-1) / n}+\cdots,
$$

where $d=m / n$ is an integer.

Substituting this into (10) for $i=1,2, \ldots, n$ and adding, we obtain

$$
\exp \left(d_{m} \omega^{d}\right)\left(\sum_{i=1}^{n} h\left(z_{i}(\omega)\right) \exp \left(d_{m-1} \omega_{i}^{(m-1) / n}+\cdots\right)\right)+\sum_{i=1}^{n} k\left(z_{i}(\omega)\right)=n f(\omega)
$$

Now, according to Lemma $2, \sum_{i=1}^{n} k\left(z_{i}(\omega)\right)$ is an entire function of order less than $d$ (since it is easy to verify that each function $k\left(z_{i}(\omega)\right)$ grows no faster than an entire function of order $d-\varepsilon_{i}$ (for some positive constant $\varepsilon_{i}$ )), and let us denote it by $T(\omega)$. It is also easy to verify that the growth of $h\left(z_{i}(\omega)\right.$ ) (for $i=1,2, \ldots, n)$ is not greater than that of an entire function of order $d-\delta_{i}$ (for some positive constant $\left.\delta_{i}\right)$. We note $\exp \left(d_{m-1} \omega_{i}^{(m-1) / n}+\cdots\right)$ also grows no faster than an entire function of order $d-\eta_{i}$ (for $i=1,2, \ldots, n$ and for some positive constant $\left.\eta_{i}\right)$, when $|\omega|$ is sufficiently large.

We rewrite identity (16) as

(17) $\sum_{i=1}^{n}\left(h\left(z_{i}(\omega)\right) \exp \left(d_{m-1} \omega_{i}^{(m-1) / n}+\cdots\right)\right)=[n f(\omega)-T(\omega)] \exp \left(-d_{m} \omega^{d}\right)$.

Then the right-hand side of (17) is an entire function. Furthermore, the left-hand side, by virtue of the above estimates, grows no faster than an entire function of order $d-\eta$ (for some positive $\eta$ ), when $|\omega|$ is sufficiently large.

Thus, we conclude that $[n f(\omega)-T(\omega)] \exp \left(-d_{m} \omega^{d}\right)$ is an entire function in $\omega$ of order less than $d$. Let us denote it by $S(\omega)$. Then we have

$$
[n f(\omega)-T(\omega)] \exp \left(-d_{m} \omega^{d}\right)=S(\omega)
$$

Consequently,

$$
f(\omega)=(S(\omega) / n) \exp \left(d_{m} \omega^{d}\right)+T(\omega) / n
$$

From this, (7) and (1), we have

$$
h(z) \exp (p(z))+k(z) \equiv(S(L(z)) / n) \exp \left(d_{m}(L(z))^{d}\right)+T(L(z)) / n .
$$

Hence

$$
h(z) \exp (p(z))-\frac{S(L(z)) \exp \left(d_{m}(L(z))^{d}\right)}{n} \equiv-k(z)+\frac{T(L(z))}{n} .
$$

Since $T(\omega), S(\omega)$ are entire functions in $\omega$ of order less than $d(=m / n)$ and $L(z)$ is entire of degree $n$, it follows that $T(L(z))$ and $S(L(z))$ are both entire functions in $z$ of order less than $m$. Thus the right-hand side of (21) is an entire function of order less than $m$. Now the left-hand side can be expressed as

$$
\exp (p(z))\left[\left(h(z)-(S(L(z)) / n) \exp \left(d_{m}(L(z))^{d}-p(z)\right)\right)\right] .
$$


We show that $h(z)-(S(L(z)) / n) \exp \left(d_{m}(L(z))^{d}-p(z)\right) \equiv U(z) \equiv 0$.

Suppose on the contrary that $U(z) \not \equiv 0$. Then $k(z)-T(L(z)) / n \neq 0$. Hence if $d_{m}(L(z))^{d}-p(z) \equiv q(z)$ has a degree $=m$, then by an application of an analog of Nevanlinna's second fundamental theorem (see e.g. [8, Theorem 2.5]) for deficient functions (here we may choose

$$
f(z)=(S(L(z)) / n) \exp \left(d_{m}(L(z))-p(z)\right),
$$

$a_{1}(z) \equiv \infty, a_{2}(z)=0, a_{3}(z)=h(z)$ in the theorem) we will get a contradiction. Alternatively, $q(z)$ has a degree less than $m$, but then this implies that the order of $U(z)$ is less than $m$. Therefore, the order of $e^{p(z)} U(z)$ is $m$ (since $k(z)-$ $T(L(z)) / n$ has order less than $m$ ) which again gives us a contradiction. Thus, we must have

$$
U(z) \equiv 0
$$

and hence, from (21)

$$
k(z)-T(L(z)) / n \equiv 0
$$

We have obtained

$$
h(z) \exp (p(z)) \equiv(S(L(z)) / n) \exp \left(d_{m}(L(z))^{d}\right)
$$

and

$$
k(z)=T(L(z)) / n \text {. }
$$

Assertions (ii) and (iii) follow from (25) and (26) respectively. The theorem is thus proved completely.

V. The proofs of Theorems 2 and 4.

The proof of Theorem 2. The "necessary" part is obvious and the "sufficient" part follows readily from Theorem 1 .

The proof of Theorem 4. Suppose that $h e^{p}+k$ under the present hypotheses is not prime; then we would have, according to Lemmas 3 and 4 , that

$$
h e^{p}+k=f(L)
$$

for some nonlinear polynomial $L$ of degree $n(n \geq 2)$ and entire $f$. By assertion (ii) of Theorem 1, we have

$$
h(z)=\mu(L) \exp \left(c[L(z)]^{d}-p(z)\right)
$$

where $\mu$ is an entire function in $L$ of order less than $m / n, c$ a constant and $d=m / n$. We note that the exponent of convergence of the zeros of $\mu(L)$ is less than the order of $h(z)$ and hence less than 1 . Therefore, we can express $\mu(z)$ as $\mu(z)=\pi(z) e^{q(z)}$, where $\pi(z)$ is an entire function of order less than 1 . Hence, we have

$$
\mu(L(z))=\pi(L(z)) e^{q(L(z))} .
$$


Substituting (32) into (31), we find that if $c[L(z)]^{d}-p(z)+q(L(z)) \not \equiv$ constant, then the order of $h$ would be at least one, a contradiction. Hence, we must conclude $p(z)=c[L(z)]^{d}+q(L(z))+c_{1}\left(c_{1}\right.$ a constant) which also can be expressed as $p(z)=q_{1}(L(z))$ for some polynomial $q_{1}$. On the other hand $k=k_{1}(L)$ by Theorem 1 . Therefore, $p$ and $k$ are not relatively $R$-polynomial prime, a contradiction. Thus equation (30) cannot hold and the theorem is proved.

In conclusion, we remark that it is not difficult to exhibit counterexamples to illustrate that the restrictions on the orders of $h$ and $k$ are necessary in all of the theorems obtained above.

Acknowledgement. The authors are indebted to Dr. Charles Osgood for his suggestions and fruitful discussions with regard to the proof of Theorem 1.

\section{REFERENCES}

1. I. N. Baker and F. Gross, Further results on factorization of entire functions, Entire Functions and Related Parts of Analysis (Proc. Sympos. Pure Math., La Jolla, Calif., 1966), Amer. Math. Soc., Providence, R. I., 1968, pp. 30-35. MR 38 \#6066.

2. R. P. Boas, Entire functions, Academic Press, New York, 1954. MR 16, 914.

3. P. Fatou, Sur l'itération des fonctions transcendantes entières, Acta Math. 47 (1926).

4. R. Goldstein, On factorisation of certain entire functions, J. London Math. Soc. (2) 2 (1970), 221-224. MR 41 \#2012.

5. , On factorisation of certain entire functions. II, Proc. London Math. Soc. (3) 22 (1971), 483-506. MR 45 \# 546.

6. - Some results on factorization of meromorphic functions, J. London Math. Soc. (2) 4 (1971), 357-364. MR 44 \#6963.

7. F. Gross, Prime entire functions, Trans. Amer. Math. Soc. 161 (1971), 219-233. MR 45 \#547.

8. - On periodic left factors of meromorphic functions, Math. Research Center, Report 69-1, NRL Report 6963, U.S. Naval Research Laboratory, Washington, D.C., 1969, 8 pp. MR 43 \#6437.

9. , Factorization of entire functions which are periodic $\bmod 8$, Indian J. Pure Appl. Math. 2 (1971).

10. F. Gross and C. C. Yang, The fix-points and factorization of meromorphic functions, Trans. Amer. Math. Soc. 168 (1972), 211-219.

11. W. K. Hayman, Meromorphic functions, Oxford Math. Monographs, Clarendon Press, Oxford, 1964. MR 29 \# 1337.

12. E. Hille, Analytic function theory. Vol. I, Introduction to Higher Math., Ginn, Boston, Mass, 1959, p. 267. MR 21 \#6415.

13. M. Ozawa, On prime entire functions. 1 Kōdai Math. Sem. Rep. 22 (1970), 301-308; II. Kōdai Math. Sem. Rep 22 (1970), 309-312. MR 42 \#4732.

14. A. Rényi and C. Rényi, Some remarks on periodic entire functions, J. Analyse Math. 14 (1965), 303-310. MR 31 \#2406.

15. J. F. Ritt, Prime and composite polynomials, Trans. Amer. Math. Soc. 23 (1922), 51-66.

16. P. C. Rosenbloom, The fix-points of entire functions, Medd. Lunds Univ. Math. Sem., Tome Suppl., (1952), 186-192. MR 14, 546.

17. C. C. Yang, On the dependence of the zeros of an entire function and its factorization, J. Math. Anal. Appl. 35 (1971), 374-381.

Department of Mathematics, University of Maryland, Baltimore County, Baltmoore, MARYLAND 21228

Mathematics Research Center, Naval Research Laboratory, Washington, D. C. 20375 